\title{
Eye movement desensitisation and reprocessing
}

\author{
M. J. MacCulloch
}

Eye movement desensitisation and reprocessing (EMDR) was described by Shapiro $(1989 a, b)$ as a new method for treating post-traumatic stress disorder (PTSD). In May 1987, while walking in the park, Shapiro noticed that her own disturbing thoughts changed then disappeared "without any conscious effort" (Shapiro, 1995) when they had been temporally paired with diagonal upward to and fro eye movements. Over the next six months Shapiro worked with approximately 70 people to develop a procedure based on the temporal pairing of distressing images and thoughts with various eye movements. Shapiro began to develop strategies to unblock stalled emotional processing, which was initiated by EMDR in non-patients. She successfully tried the method on a Vietnam veteran suffering from severe PTSD and then embarked upon a trial of EMDR on a mixed group of victims of rape, molestation and Vietnam combat trauma. Initially, EMDR achieved wide recognition as a new breakthrough treatment for PTSD. This was, in part, because of very positive early reports (e.g. Wolpe \& Abrams, 1991), but also because the EMDR effect appeared to occur with unprecedented speed, often in cases of PTSD that had previously resisted treatment by many other methods over a long period.

\section{Method}

Eye movement desensitisation and reprocessing is taught as an eight-phase treatment method (see Box 1) which includes history-taking, patient preparation, assessment, desensitisation (counterconditioning), installation, body scan, closure and re-evaluation. It is based on the premise that contiguous pairings of therapist-directed eye movements (or bilateral shift of attention in other modalities such as sound or body taps) with traumatic memories allows re-evaluation and reclassification of those memories. After successful EMDR treatment, probes of treated memories fail to produce distress, maladaptive behaviour, feelings or attitudes. The eight phases of the method are designed as a protocol to ensure that all the preexisting relevant and cross-associated traumatic memories are identified, reproduced, controlled and paired with the eye movement procedure in a safe manner. The stages of EMDR treatment are as follows.

\section{History}

The creation of therapeutic bonding is a crucial part of history-taking because EMDR is intrusive, powerful and anxiety-provoking. The history elicits

\section{Box 1. Eight stages of EMDR}

History
Preparation
Target assessment
Desensitisation
Installation
Body Scan
Closure
Re-evaluation

History

Preparation

Target assessment

Desensitisation

Installation

Closure

Re-evaluation

Malcolm MacCulloch is Professor of Forensic Psychiatry at Cardiff University and the South Wales Forensic Psychiatry Service at the Caswell Clinic (Glanrhyd Hospital, Bridgend, Mid Glamorgan CF31 4LN). He was formerly a child psychiatrist, and worked for the Department of Health and Social Security before becoming Medical Director of Ashworth North Special Hospital. His research interests include aspects of antisocial personality disorder and aspects of post-traumatic stress disorder and its treatment by eye movement desensitisation and reprocessing. 
a clear view of the traumatic and relevant antecedent events for both the therapist and the victim. Trauma memories encode all sense modalities, it is important that dissociative phenomena and amnesias are identified in order to allow full description of the traumatic episode. The patient's ability to work with the therapist and to deal with fresh, partly forgotten traumatic memories must be established.

\section{Preparation}

Patients are taught that avoidance maintains PTSD, and that the process of EMDR restarts emotional processing. Patients are taught self-control, including methods of reducing ambient anxiety during EMDR. During preparation, patients are instructed to expect vivid re-experiencing of memories in all modalities, and reassured that they have unconditional support from the therapist who will maintain and 'ground' them in the therapeutic present, throughout their experiences of recall. A considerable time may be required to develop the therapeutic relationship to a point where the patient can handle the emotions that accompany fresh emotional processing.

\section{Target assessment}

The target traumatic memory is explored and defined in detail. The memory may be stored in iconic fragments and it is traumatic both to recall and to utilise in the therapeutic process. The patient is trained to assess the negative emotional impact of the memory in terms of the Subjective Units of Disturbance (SUD) scale (Wolpe, 1990). SUDs range from 0 to 10 , where 10 is the "worst emotion of my life". The effects of different memories are ordered using SUDs in order to select therapeutic targets and to assess the effect of the ongoing EMDR on each aspect of trauma memory including associated memories and body feelings. The patients are helped to express verbal descriptions of how they feel when they are asked to recall previously agreed memories prior to the start of the EMDR treatment. These phrases are self-referencing negative beliefs such as, 'it was my fault' which, before EMDR, represent how the patient views the remembered event. So long as that negative view is maintained and triggers the associated negative affect and unpleasant somatic sensation, the memory and its effects are unprocessed and will continue to be a source of distress. The essence of the desensitisation component of EMDR treatment is to associate recollections of the sensory images, verbal descriptions, bodily sensations and affect (which all relate to a particular trauma), with the de-arousing properties of eye movement.
The EMDR process alters the patient's evaluation of his or her memories, and the changes in those evaluations are immediately indicated by a fall in the SUD ratings. Prior to the start of EMDR patients are helped to define a desired state in respect to their memories of the trauma. For example, a desired positive cognition would be formulated as 'I did the best that I could', instead of the cognition 'it was my fault'. Additionally, the truth of that positive statement is assessed before treatment by the use of a seven-point scale referred to as Validity of Cognition (VoC; Shapiro, 1989b). In effect, the patient is asked to indicate how true the desired positive cognition is before treatment on a scale where 1 is 'completely false' and 7 is 'completely true'. A second component of EMDR, referred to as 'installation', associates the EMDR effect with positive inner-self statements related to recall of the original trauma images at a stage in the treatment when recall of trauma images has become neutral, that is, evokes SUDs of less than two.

\section{Desensitisation}

In EMDR, desensitisation refers to the process in which the emotional effect of trauma memories is altered from being aversive to being neutral. In trauma cases that have not been resolved, by either a natural process of emotional processing, or by other therapeutic methods, patients are left in a state in which dysfunctional anticipatory defence mechanisms persist. In this situation abnormal fears are indicated by the persistence of anxiety resulting from the unprovoked return and incubation of fear.

The eye movement effect is contiguously paired in EMDR with the therapist's directed evocation of: "the picture which represents the worst part of the incident and the words that go best with that picture and which express your negative belief about yourself 'now' and the bodily feelings and emotions that accompany the memory image".

The patient is instructed to generate the previously agreed image, rehearse the negative statement and must attend to negative bodily sensations and then to follow the therapist's finger moving to and fro at 120 saccades a minute about $35 \mathrm{~cm}$ in front of his or her face.

The patient is reassured about his or her performance and progress. Emotional abreaction commonly occurs within a few cycles of the start of eye movement; patients show features of fear and anxiety, they report vivid video-like memories of the trauma scene. Eye movement is continued whenever and as long as the abreaction takes place. Various techniques (beyond the scope of this article) are used to control unbearable levels of anxiety (Shapiro, 1995) and where processing ceases (as indicated by 
SUDs ceasing to fall) other techniques (such as changing the angle of the eye movement) are used to unblock the stalled processing. The EMDR is continued until all the negative emotions associated with the original memories are rated as having SUDs of two or less; that is, they no longer evoke negative feelings and at this point installation is commenced.

\section{Installation}

The patient is asked to recall the trauma and to hold in mind the (preferred) positive cognition, such as, 'I did the best that I could' and is then treated with further repeated sets of eye movements. The validity of the positive cognition is checked by the question, "on a scale of 1-7, how true of the memory is the statement: 'I did the best I could'". When the VoC score rises after repeated eye movements to a value of six or seven and subsequently does not increase with further repeated sets of eye movements then the treatment proceeds to the next stage.

\section{Body scan}

The patient is told to close their eyes and to focus on bodily sensations when they recall the original traumatic incident. If any negative body sensation is reported, then eye movement is repeated associated with the memory and the body perception until no further negative body sensation is reported.

The body scan and eye movement part of the procedure causes the remembered negative somatic sensations (such as pain; see Hassard, 1995) to be paired with the eye movement effect until the memory of the negative body sensation is abolished.

\section{Closure}

Patients are told to expect further between-session processing, that is, repeated changing and fresh emotional memories. They are asked to keep a note of any new material that emerges between sessions and before the end of each session they are taken through a relaxation exercise that was taught as part of the preparation for EMDR treatment.

\section{Re-evaluation}

Re-evaluation takes place at the start of each new EMDR session and involves decisions about the need for further EMDR treatment. When the patient's between-treatment symptom log ceases to reveal the persistence of the negative effects of old trauma memories or the emergence of new difficulties encountered in daily life, then the EMDR can be terminated. Continued follow-up, however, is important, not least because of the patient's need to know that the 'therapeutic door' remains open. It is important not to assume that every possible unconscious dysfunction has been resolved within a given number of eye movement sessions. The EMDR protocol can be thought of as comprising three main stages: (a) working on past traumas; (b) working on current stimuli which continue to elicit disturbing and dysfunctional material; and (c) focusing on the patient's ability to make new choices in the future. This third process relies upon the identification and 'reprocessing' of anticipatory fears. The third stage also includes education, modeling and anticipatory conjectures which consider significant people and significant situations that may be encountered in the future.

\section{Comparisons with other treatments for PTSD}

The efficacy of treatments for PTSD is a psychological matter about which there is increasing concern, for example, Solomon et al (1992) provide a wide-ranging review of a variety of treatments for PTSD which considered 225 reports of which only 11 were randomised trials. They concluded that: "much more research is needed, however, before any strong conclusions can be drawn about the efficacy of pharmacotherapies for PTSD". In considering six studies which examined the efficacy of behavioural techniques they cautioned that severe complications have been reported with the use of flooding, although that treatment when compared with stress inoculation treatment was superior after three months' follow-up. Solomon et al (1992) did not review EMDR treatments.

Shalev et al (1996) also reviewed treatments for PTSD. In addition to a review of 26 pharmacological studies they also considered the psychological approach to the treatment of PTSD and reviewed 39 treatments that included flooding, relaxation, therapeutic exposure, desensitisation, image habituation, military training, helicopter ride therapy, cognitive processing, dynamic psychotherapy, anger control, group therapy, hypnosis, rehabilitation and various combined approaches. Like Solomon et al (1992), no consideration of EMDR was included in the review, Shalev et al do, however, provide an informative section on the aetiology of PTSD which is conceptualised as a combination of classical and operant conditioning. The resistance to extinction is explained by invoking the concept 
of operant conditioning in which the avoidance of a variety of internal and external cues associated with the trauma is rewarded by reduction in distress, which in turn expands the avoidant behaviour to secondary and tertiary cues. Shalev et al (1996) conclude their review with a number of interesting and important statements about PTSD. None of the studies reviewed by Shalev $e$ al was found to have achieved durable remission in chronic PTSD, leading them to conclude that the disorder is partly unresponsive to any form of treatment, although many treatments produce a degree of improvement. Recurrent chronic recollections represent an:

\footnotetext{
"obsessive, repetitive dysfunction of memory, in respect of which attempts to explore, elaborate and work through these memories at later stages often aggravate rather than cure."
}

\begin{abstract}
Also:
"conditioned fear responses may, similarly, become indelible and thereby inaccessible to eradication through desensitisation or flooding."
\end{abstract}

\section{Finally:}

"one should remember that, to some extent, the imprint (of the trauma), is indelible and hence immutable by current methods."

The omission by these otherwise satisfactory reviews of any reference to EMDR as a treatment for PTSD is both surprising and disappointing. This perhaps reflects a degree of ambivalence towards EMDR which is referred to by Boudewyns \& Hyer, (1996) as possibly arising from Shapiro's (1989b) enthusiastic claims for her treatment and her perceived control of those who may practice it. Eye movement desensitisation and reprocessing remains controversial (Herbert \& Meuser, 1992; Lohr et al,1992; Page \& Crino, 1993, Acierno et al, 1994), but there are increasing numbers of reports of its efficacy from respected authors (Spector \& Huthwaite, 1993; Vaughan et al, 1994a,b; Wilson et al, 1996; Van de Kolk et al 1996; Rothbaum, 1997; Carlson et al, 1998; Scheck et al, 1998).

The first reports of the efficacy of EMDR were followed by a number of papers that were produced by workers who had not been trained in EMDR. These articles reported equivocal results. Posttraumatic stress disorder itself is a complex disorder, the chronic form of which is extremely resistant to treatment. The possibility arises that there are two forms of PTSD: extinguishing PTSD, which is the most common, and non-extinguishing PTSD, which is rarer but which comprises the bulk of the cases that cause therapeutic concern. If this suggestion is correct, then the equivocal findings concerning the EMDR treatment of PTSD can be explained by a combination of factors as follows.
First, it is probably the case that experimental groups of PTSD sufferers in which EMDR was compared with other treatments were heterogeneous. That is, they contained unspecified mixtures of cases, some of which were fixed and immutable to most treatments including badly administered EMDR, and others that were capable of emotional processing by a variety of methods including natural emotional processing.

Second, badly administered EMDR is likely to be composed of elements of exposure and cognitive restructuring techniques. This can be expected to work with some (extinguishing) cases of PTSD and, in addition, a significant number of workers will have attempted to treat non-extinguishing PTSD using a faulty EMDR technique that failed to evoke the forced relaxation response (Wilson et al, 1996). This state of affairs probably accounts for the conflicting results of a number of trials of EMDR which sought to emulate the high levels of success that were reported in the early publications by the originator of the technique (Shapiro, 1989a,b).

\section{Brief appraisal of EMDR}

(a) Eye movement desensitisation and reprocessing depends upon a cerebral reflex that counteracts fear responses (MacCulloch \& Feldman, 1996).

(b) The clinical procedures of EMDR override previously conditioned fears by linking positive (safe) feelings to the fear-provoking conditioned stimulus.

(c) It is generally accepted that there is an 'n-shaped' relationship between arousal and efficiency (Malmo, 1959). Specifically, performance falls as arousal rises and in PTSD, beyond a certain threshold, some tasks cease abruptly (i.e. emotional processing).

(d) The immediate consequence of EMDR is to allow emotional processing because it moves the subject's position on the arousal/ efficiency curve to a point at which processing can begin. Emotional and cognitive processing can proceed without automatic inner avoidance.

\section{Research findings}

(a) Changes in the subject's perception of the conditioned stimulus conditions change very rapidly (Kleinknecht \& Morgan, 1992; Armstrong \& Vaughan, 1996). 
Box 2. PTSD and EMDR

PTSD is a common result of life-threatening trauma

Most cases get better/respond to treatment

The persistent minority are very resistant to most treatments

EMDR is a unique, complex psychological technique based upon induced de-arousal

Physiological de-arousal has been demonstrated over the course of EMDR

Pre- and post-treatment neuro-imaging studies have shown changes in PTSD cases treated by EMDR

Successful EMDR works very quickly and has been successful in treating PTSD cases previously resistant to other forms of treatment

(b) The EMDR effect is accompanied by de-arousal (Wilson et al, 1996).

(c) The technique is successful in cases where other therapeutic methods have failed (Vaughan \& Tarrier, 1992; Vaughan et al, 1994a).

(d) Van der Kolk et al (1996) report cerebral imaging data and conclude that recovery from PTSD depends on higher brain functions that override input from limbic structures (i.e. amygdala).

\section{Discussion and conclusions}

Recent studies have provided convincing evidence for the effectiveness of EMDR, however, the problem of inconsistent results has remained. The present appraisal lists some recent studies that account for the previous inconsistencies in the literature on EMDR. A summary of PTSD and EMDR is given in Box 2.

As a cautionary note, at this stage in the development of our understanding of EMDR it would be wise for those who contemplate its use to undergo formal training. Also it would be prudent, for therapists to confine themselves to the treatment of cases of conditioned aversion characterised by some, if not all, of the features of PTSD.

\section{References}

Acierno, R., Tremont, G., Last, C., et al (1994) Tripartite assessment of the efficacy of eye-movement desensitisation in a multi-phobic patient. Journal of Anxiety Disorders, 8, 259-276.

Armstrong, M. S. \& Vaughan, K. (1996) An Orienting response model of eye movement desensitisation. Journal of Behaviour Therapy and Experimental Psychiatry, 27, 21-32.

Boudewyns, P. A. \& Hyer, L. A. (1996) Eye Movement desensitisation and reprocessing (EMDR) as treatment for post-traumatic stress disorder (PTSD). Clinical Psychology and Psychotherapy, 3, 185-195.

Carlson, G. C., Chemtob, C. M., Rusnak, K., et al (1998). Eye movement desensitisation and reprocessing (EMDR) treatment for combat-related post-traumatic stress disorder. Journal of Traumatic Stress, 11, 3-24.

Hassard, A. (1995) Investigation of eye movement desensitisation in pain clinic patients. Behavioral and Cognitive Psychotherapy, 23, 177-185.

Herbert, J. D. \& Mueser, K. (1992) Eye movement desensitisation: a critique of the evidence. Journal of Behaviour Therapy and Experimental Psychiatry, 23, 169-174.

Kleinknecht, R. \& Morgan, M. (1992) Treatment of posttraumatic stress disorder with eye movement desensitisation and reprocessing. Journal of Behaviour Therapy and Experimental Psychiatry, 23, 43-49.

Lohr, J. M., Kleinknecht, R. A., Conley, A. T., et al (1992). A methodological critique of the current status of eye movement desensitisation. Journal of Behaviour Therapy and Experimental Psychiatry, 23, 159-167.

MacCulloch, M. J. \& Feldman, M. P. (1996) Eye movement desensitisation treatment utilizes the positive visceral element of the investigatory reflex to inhibit the memories of post-traumatic stress disorder: a theoretical analysis. British Journal of Psychiatry, 169, 571-579.

Malmo, R. B. (1959) Activation: a neuropsychological dimension. Psychological Review, 66, 367-386.

Page, A. \& Crino, R. (1993) Eye-movement desensitisation: a simple treatment for post-traumatic stress disorder? Australian and New Zealand Journal of Psychiatry, 27, 288-293.

Rothbaum, B. O. (1997) A controlled study of eye movement desensitisation and reprocessing in the treatment of posttraumatic stress disordered sexual assault victims. Bulletin of the Menninger Clinic, 61, 317-334.

Scheck, M. M., Schaeffer, J. A. \& Gillette, C. (1998) Brief psychological intervention with traumatized young women: the efficacy of eye movement desensitisation and reprocessing. Journal of Traumatic Stress, 11, 25-44.

Shalev, A. Y., Bonne, O. \& Eth, S. (1996) Treatment of posttraumatic stress disorder: A review. Psychosomatic Medicine, 58, 165-182.

Shapiro, F. (1989a) Efficacy of the eye movement desensitisation procedure in the treatment of traumatic memories. Journal of Traumatic Stress, 2, 199-223.

- (1989b) Eye movement desensitisation: a new treatment for post-traumatic stress disorder. Journal of Behaviour Therapy and Experimental Psychiatry, 20, 211-217.

- (1995) Eye Movement Desensitisation and Reprocessing: Basic Principles, Protocols and Procedures. New York: Guilford.

Solomon, S. D., Gerrity, E. T. \& Muff, A. M. (1992) Efficacy of treatments for post-traumatic stress disorder: an empirical review. Journal of the American Medical Association, 268, 633-638.

Spector, J. \& Huthwaite, M. (1993) Eye-movement desensitisation to overcome post-traumatic stress disorder. British Journal of Psychiatry, 163, 106-108.

Van der Kolk, B.A., Burbridge, J. A. \& Suzuki, J. (1996) The psychobiology of traumatic memory. Clinical implications of neuroimaging studies. Annals of the New York Academy of Sciences, 821, 99-113.

Vaughan, K. \& Tarrier, N. (1992) The use of image habituation training with post-traumatic stress disorders. British Journal of Psychiatry, 161, 658-664.

- Armstrong, M., Gold, R., et al (1994a) A trial of eye movement desensitisation compared to image habituation training and applied muscle relaxation in post-traumatic stress disorder. Journal of Behaviour Therapy and Experimental Psychiatry, 25, 283-291. 
-, Wiese, M., Gold, R., et al (1994b) Eye-movement desensitisation symptom change in post-traumatic stress disorder. British Journal of Psychiatry, 164, 533-541.

Wilson, D. L. Silver, S. M. \& Covi, W. G. (1996) Eye movement desensitisation and reprocessing: effectiveness and autonomic correlates. Journal of Behaviour Therapy and Experimental Psychiatry, 27, 219-229.

Wolpe, J. (1990) The Practice of Behaviour Therapy (4th edn). New York: Pergamon Press.

— \& Abrams, J. (1991) Post-traumatic stress disorder overcome by eye movement desensitisation: a case report. Journal of Behaviour Therapy and Experimental Psychiatry, 22, 39-43.

\section{Multiple choice questions}

\section{PTSD:}
a is a rare condition
b nearly always responds to some kind of treatment
c is the result of aversive conditioning
d requires emotional processing for its resolution
e can be made worse by flooding.

\section{EMDR:}

a is taught in eight stages

b can be successful in cases of chronic PTSD

c is incompatible with other methods of treatment

d cannot make a patient worse

e only works for PTSD.

3. In EMDR:

a changes in cerebral blood flow have been demonstrated

b there is no evidence of physiological dearousal

c the orienting reflex is thought to be involved in the therapeutic effect

d rapport is not essential with the patient

e therapeutic changes usually take place very quickly.
4. In the course of EMDR therapy:

a never talk or allow the patient to talk and do not offer encouragement

b if the patient appears stuck, the therapist can change the direction or the speed of the eye movement

c have the patient keep notes of distressing thoughts or feelings between sessions

d SUDs are on a scale of $0-10$

e positive cognition scores are on a scale of 1-7.

5. In traumatic stress:

a traumatic memories are chiefly visual

b environmental signals can change their meaning

c there is a linear relationship between arousal and cerebral efficiency

d EMDR was originally based on a systematic theory

e successful EMDR leads to emotional processing of traumatic memories.

\section{MCQ answers}

\begin{tabular}{|c|c|c|c|c|}
\hline 1 & 2 & 3 & 4 & 5 \\
\hline a F & a $T$ & a $T$ & a $F$ & a F \\
\hline b F & b $T$ & b $F$ & b $\mathrm{T}$ & b $\mathrm{T}$ \\
\hline c $\mathrm{T}$ & c $\mathbf{F}$ & c $\mathrm{T}$ & c $\mathrm{T}$ & c $F$ \\
\hline d $T$ & d F & d F & d $T$ & d F \\
\hline $\mathrm{T}$ & e $F$ & e $T$ & e $T$ & e $\mathrm{T}$ \\
\hline
\end{tabular}

